\title{
Univalence Conditions of Two New Integral Operators on P-Valent Functions
}

\author{
Nguyen Van Tuan ${ }^{1} \&$ Daniel Breaz ${ }^{2}$ \\ ${ }^{1}$ Department of Mathematics, University of Pitesti, Targul din Vale Str., No.1, 110040, Pitesti, Arges, Romania \\ 2 "1 Decembrie" University of Alba Iulia, Romania, N. Iorga Str., No. 11-13,510009, Alba Iulia, Romania
}

Correspondence: Nguyen Van Tuan, Department of Mathematics, University of Pitesti, Targul din Vale Str., No.1, 110040, Pitesti, Arges, Romania

Received: September 2, 2018 Accepted: February 22, 2019 Online Published: March 4, 2019

doi:10.5539/jmr.v11n2p63

URL: https://doi.org/10.5539/jmr.v11n2p63

\section{Abstract}

For analytic functions in the open unit disk $\mathcal{U}$, we define two new general integral operators. The main object of the this paper is to study these two new integral operators and to determine some sufficient conditions for general $p$-valent integral operator to be $p$-th power of a univalent functions.

Keywords: analytic functions, univalent functions, integral operators, sufficient conditions, $p$-valent integral operators

2010 Mathematics Subject Classification: Primary 30C45; Secondary 30C75

\section{Introduction}

Let $\mathcal{A}_{p}$ be the class of functions of the form:

$$
f(z)=z^{p}+\sum_{k=p+1}^{\infty} a_{k} z^{k}, z \in \mathcal{U}, p \in \mathbb{N}^{*}=\{1,2, \ldots, n\}
$$

which are analytic and $p$-valent in the unit disk $\mathcal{U}=\{z:|z|<1\}$.

A function $f \in \mathcal{A}_{p}$ is called $p$-valent starlike of order $\gamma$ if $f(z)$ satisfies

$$
\operatorname{Re}\left(\frac{z f^{\prime}(z)}{f(z)}\right)>\gamma, z \in \mathcal{U}
$$

$0 \leq \gamma<p, p \in \mathbb{N}^{*}$. We denote by $\mathcal{S}_{p}^{*}(\gamma)$ the class of all such functions.

A function $f \in \mathcal{A}_{p}$ is in the class $\mathcal{S}_{p}^{*}(\gamma), 0 \leq \gamma<p, p \in \mathbb{N}^{*}$ if it satisfies the condition

$$
\left|\frac{z f^{\prime}(z)}{f(z)}-p\right|<p-\gamma, z \in \mathcal{U} .
$$

A function $f \in \mathcal{A}_{p}$ is called p-valent convex of order $\gamma$ if $f(z)$ satisfies

$$
\operatorname{Re}\left(1+\frac{z f^{\prime \prime}(z)}{f^{\prime}(z)}\right)>\gamma, z \in \mathcal{U},
$$

$0 \leq \gamma<p, p \in \mathbb{N}^{*}$. By $C_{p}(\gamma)$, we denote the class of all p-valent convex function of order $\gamma$. From (1.2) and (1.4), Çağlar showed us that:

$$
f(z) \in C_{p}(\gamma) \Leftrightarrow \frac{z f^{\prime}(z)}{p} \in \mathcal{S}_{p}^{*}(\gamma) .
$$

A function $f \in \mathcal{A}_{p}$ is in the class $C_{p}(\gamma), 0 \leq \gamma<p, p \in \mathbb{N}^{*}$ if it satisfies the condition

$$
\left|\frac{z f^{\prime \prime}(z)}{f^{\prime}(z)}-(p-1)\right|<p-\gamma, z \in \mathcal{U} .
$$

Now we define two new general $p$-valent integral operators. 
The first new $p$-valent integral operator has the following form:

$$
J_{\alpha_{i}, \beta}^{p}(z)=\left\{\beta p \int_{0}^{z} t^{\beta p-1} \prod_{i=1}^{\infty}\left(\frac{f_{i}(t)}{t^{p}}\right)^{1-\frac{1}{\alpha_{i}}} d t\right\}^{\frac{1}{\beta}}
$$

where the functions $f_{i} \in \mathcal{A}_{p}, i=1,2, \ldots, n$, and the paramentes $\beta$ and $\alpha_{i}, \alpha_{i} \neq 0, \operatorname{Re}(\beta)>0$ for all $i=1,2, \ldots, n$ are complex numbers such that the integral operators in (1.7).

The second new $p$-valent integral operator has the form

$$
I_{\alpha_{i}, \beta}^{p}(z)=\left\{\beta p \int_{0}^{z} t^{\beta p-1} \prod_{i=1}^{n}\left(\frac{f_{i}(t)}{t^{p}}\right)^{1-\frac{1}{\alpha_{i}}}\left(\frac{g_{i}^{\prime}(t)}{p t^{p-1}}\right)^{\frac{1}{\alpha_{i}}} d t\right\}^{\frac{1}{\beta}}
$$

where the functions $f_{i}, g_{i} \in \mathcal{A}_{p}, i=1,2, \ldots, n$, and the paramentes $\beta$ and $\alpha_{i}, \alpha_{i} \neq 0, \operatorname{Re}(\beta)>0$ for all $i=1,2, \ldots, n$ are complex numbers such that the integral operators in (1.8). Hallenbeck and Livingston defined $p$-subordination chains method and they obtained some results for $f \in \mathcal{A}_{p}$ to be the $p$-th power of a univalent functions in $\mathcal{U}$.

Theorem 1.1. Let $f \in \mathcal{A}_{p}$ and $\alpha$ complex number such that $\operatorname{Re} \alpha>0$ and

$$
\frac{1-|z|^{2 p \operatorname{Re} \alpha}}{\operatorname{Re} \alpha}\left|\frac{z f^{\prime \prime}(z)}{f^{\prime}(z)}-(p-1)\right| \leq p
$$

is true for all $z \in \mathcal{U}$, then the integral operator

$$
\mathcal{H}_{\alpha}(z)=\left\{\alpha \int_{0}^{z} t^{p(\alpha-1)} f^{\prime}(t) d t\right\}^{\frac{1}{\alpha}}
$$

is the p-th power of a univalent function in $\mathcal{U}$ where the principal branch is considered.

\section{Main Results}

Firstly, we obtain sufficient conditions for $p$-valent integral operator defined by (1.7), to be the $p$-th power of a univalent function in $\mathcal{U}$.

Theorem 2.1. Let the function $f_{i} \in \mathcal{S}_{p}^{*}\left(\gamma_{i}\right), 0 \leq \gamma_{i}<p, p \in \mathbb{N}^{*}, i=1,2, \ldots, n$. If $\beta, \alpha_{i} \in \mathbb{C}, \alpha_{i} \neq 0, i=1,2, \ldots, n$ and

$$
p \operatorname{Re} \beta \geq 1-p+\sum_{i=1}^{n} \frac{\left|\alpha_{i}-1\right|\left(p-\gamma_{i}\right)}{\left|\alpha_{i}\right|}
$$

then the integral operator $J_{\alpha_{i}, \beta}^{p}$ defined by (1.7) is the p-th power of a univalent function in $\mathcal{U}$.

Proof. We define the function

$$
h(z)=\int_{o}^{z} \prod_{i=1}^{n}\left(\frac{f_{i}(t)}{t^{p}}\right)^{1-\frac{1}{\alpha_{i}}} d t .
$$

It is easy to see that

$$
h^{\prime}(z)=\prod_{i=1}^{n}\left(\frac{f_{i}(z)}{z^{p}}\right)^{1-\frac{1}{\alpha_{i}}} .
$$

Differentiating (2.3) logarithmically and multiplying by z, we get

$$
\frac{z h^{\prime \prime}(z)}{h^{\prime}(z)}=\sum_{i=1}^{n}\left(1-\frac{1}{\alpha_{i}}\right)\left(\frac{z f_{i}^{\prime}(z)}{f_{i}(z)}-p\right) \text {. }
$$

Thus, we obtain 


$$
\begin{aligned}
& \frac{1-|z|^{2 p \operatorname{Re} \beta}}{\operatorname{Re} \beta}\left|1-p+\frac{z h^{\prime \prime}(z)}{h^{\prime}(z)}\right|=\frac{1-|z|^{2 p \operatorname{Re} \beta}}{\operatorname{Re} \beta}\left|1-p+\sum_{i=1}^{n}\left(1-\frac{1}{\alpha_{i}}\right)\left(\frac{z f_{i}^{\prime}(z)}{f_{i}(z)}-p\right)\right| \\
& \leq \frac{1-|z|^{2 p \operatorname{Re} \beta}}{\operatorname{Re} \beta}\left[1-p+\sum_{i=1}^{n}\left|1-\frac{1}{\alpha_{i}}\right|\left|\frac{z f_{i}^{\prime}(z)}{f_{i}(z)}-p\right|\right] \\
& \leq \frac{1-|z|^{2 p \operatorname{Re} \beta}}{\operatorname{Re} \beta}\left[\frac{\left|\alpha_{i}-1\right|}{\left|\alpha_{i}\right|}\left|\frac{z f_{i}^{\prime}(z)}{f_{i}(z)}-p\right|\right] \\
& \leq \frac{1}{\operatorname{Re} \beta}\left[1-p+\sum_{i=1}^{n} \frac{\left|\alpha_{i}-1\right|\left(p-\gamma_{i}\right)}{\left|\alpha_{i}\right|}\right] .
\end{aligned}
$$

From the hypothesis of the Theorem 2.1, we get:

$$
\begin{aligned}
& \frac{1-|z|^{2 p \operatorname{Re} \beta}}{\operatorname{Re} \beta}\left|1-p+\frac{z h^{\prime \prime}(z)}{h^{\prime}(z)}\right| \leq \\
& \leq \frac{1}{\operatorname{Re} \beta}\left[1-p+\sum_{i=1}^{n} \frac{\left|\alpha_{i}-1\right|\left(p-\gamma_{i}\right)}{\left|\alpha_{i}\right|}\right] \leq p .
\end{aligned}
$$

Applying Theorem 1.1, we get the integral operator $J_{\alpha_{i}, \beta}^{p}$ defined by (1.7) is the $p$-th power of a univalent function in $\mathcal{u}$.

Letting $\beta=n=1, \alpha_{1}=\alpha, \gamma_{1}=\gamma$ and $f_{1}=f$ in Theorem 2.1, we obtain the following corollary.

Corollary 2.1. Let the function $f \in \mathcal{S}_{p}^{*}(\gamma), 0 \leq \gamma<p, p \in \mathbb{N}^{*}$. If $\alpha \in \mathbb{C}, \alpha \neq-1, \alpha \neq \frac{1}{3}$ and

$$
p \geq \frac{|\alpha|-\gamma|\alpha-1|}{2|\alpha|-|\alpha-1|}
$$

then the integral operator

$$
J_{\alpha, 1}^{p}(z)=J_{\alpha}^{p}(z)=\int_{0}^{z} p t^{p-1}\left(\frac{f(t)}{t^{p}}\right)^{1-\frac{1}{\alpha}} d t
$$

is the p-th power of a univalent function in $\mathcal{U}$.

Letting $\beta=n=1, \alpha_{1}=\alpha=1, \gamma_{1}=\gamma$ and $f_{1}=f$ in Theorem 2.1, we obtain following corollary.

Corollary 2.2. Let the function $f \in \mathcal{S}_{p}^{*}(\gamma), 0 \leq \gamma<p, p \in \mathbb{N}^{*}$, then the integral operator

$$
J_{1,1}^{p}(z)=J^{p}(z)=p \int_{0}^{z} t^{p-1} d t
$$

is the p-th power of a univalent function in $\mathcal{U}$.

In the next theorem, we derive another sufficient condition for $p$-valent integral operator defined by (1.8), to be the $p$-th power of a univalent function in $\mathcal{U}$.

Theorem 2.2. Let the functions $f_{i} \in \mathcal{S}_{p}^{*}\left(\gamma_{i}\right)$ and $g_{i} \in \mathcal{C}_{p}\left(\gamma_{i}\right), 0 \leq \gamma_{i}<p, p \in \mathbb{N}^{*}, i=1,2, \ldots, n$. If $\beta, \alpha_{i} \in \mathbb{C}, \alpha \neq 0$, $i=1,2, \ldots, n$ and

$$
p \operatorname{Re} \beta \geq 1-p+\sum_{i=1}^{n} \frac{\left|\alpha_{i}-1\right|+1}{\left|\alpha_{i}\right|}\left(p-\gamma_{i}\right)
$$

then the integral operator $I_{\alpha_{i}, \beta}^{p}$ defined by (1.8) is the p-th power of a univalent function in $\mathcal{U}$.

Proof. We define

$$
h(z)=\int_{0}^{z} \prod_{i=1}^{n}\left(\frac{f_{i}(t)}{t^{p}}\right)^{1-\frac{1}{\alpha_{i}}}\left(\frac{g_{i}^{\prime}(t)}{p t^{p-1}}\right)^{\frac{1}{\alpha_{i}}} d t .
$$


It is easy to see that

$$
h^{\prime}(z)=\prod_{i=1}^{n}\left(\frac{f_{i}(z)}{z^{p}}\right)^{1-\frac{1}{\alpha_{i}}}\left(\frac{g_{i}^{\prime}(z)}{p z^{p-1}}\right)^{\frac{1}{\alpha_{i}}} .
$$

Differentiating (2.12) logarithmically and multiplying by $z$, we obtain

$$
\frac{z h^{\prime \prime}(z)}{h^{\prime}(z)}=\sum_{i=1}^{n}\left[\left(1-\frac{1}{\alpha_{i}}\right)\left(\frac{z f_{i}^{\prime}(z)}{f_{i}(z)}-p\right)+\frac{1}{\alpha_{i}}\left(\frac{z g^{\prime \prime}(z)}{g_{i}^{\prime}(z)}-(p-1)\right)\right] .
$$

Thus, we get

$$
\begin{aligned}
& \frac{1-|z|^{2 p \operatorname{Re} \beta}}{\operatorname{Re} \beta}\left|1-p+\frac{z h^{\prime \prime}(z)}{h^{\prime}(z)}\right|= \\
& =\frac{1-|z|^{2 p \operatorname{Re} \beta}}{\operatorname{Re} \beta}\left|1-p+\sum_{i=1}^{n}\left[\left(1-\frac{1}{\alpha_{i}}\right)\left(\frac{z f_{i}^{\prime}(z)}{f_{i}(z)}-p\right)+\frac{1}{\alpha_{i}}\left(\frac{z g_{i}^{\prime \prime}(z)}{g_{i}^{\prime}(z)}-(p-1)\right)\right]\right| \\
& \leq \frac{1-|z|^{2 p \operatorname{Re} \beta}}{\operatorname{Re} \beta}\left[1-p+\sum_{i=1}^{n}\left(\frac{\left|\alpha_{i}-1\right|}{\left|\alpha_{i}\right|}\left|\frac{z f_{i}^{\prime}(z)}{f_{i}(z)}-p\right|+\frac{1}{\left|\alpha_{i}\right|}\left|\frac{z g_{i}^{\prime \prime}(z)}{g^{\prime}(z)}-(p-1)\right|\right)\right] \\
& \leq \frac{1-|z|^{2 p \operatorname{Re} \beta}}{\operatorname{Re} \beta}\left[1-p+\sum_{i=1}^{n}\left(\frac{\left|\alpha_{i}-1\right|}{\left|\alpha_{i}\right|}\left(p-\gamma_{i}\right)+\frac{1}{\left|\alpha_{i}\right|}\left(p-\gamma_{i}\right)\right)\right] \\
& \leq \frac{1}{\operatorname{Re} \beta}\left[1-p+\sum_{i=1}^{n} \frac{\left|\alpha_{i}-1\right|+1}{\left|\alpha_{i}\right|}\left(p-\gamma_{i}\right)\right] .
\end{aligned}
$$

From the hypothesis of the Theorem 2.2, we get

$$
\begin{aligned}
& \frac{1-|z|^{2 p \operatorname{Re} \beta}}{\operatorname{Re} \beta}\left|1-p+\frac{z h^{\prime \prime}(z)}{h^{\prime}(z)}\right| \\
& \leq \frac{1}{\operatorname{Re} \beta}\left[1-p+\sum_{i=1}^{n} \frac{\left|\alpha_{i}-1\right|+1}{\left|\alpha_{i}\right|}\left(p-\gamma_{i}\right)\right] \leq p .
\end{aligned}
$$

Applying Theorem 1.1, we get the integral operator $I_{\alpha_{i}, \beta}^{p}$ defined by (1.8) is the $p$-th power of a univalent function in $\mathcal{U}$.

Letting $\beta=n=1, \gamma_{1}=\gamma, \alpha_{1}=\alpha, f_{1}=f$ and $g_{1}=g$ in Theorem 2.2, we get the following corollary.

Corollary 2.3. Let the functions $f \in \mathcal{S}_{p}^{*}(\gamma)$, and $g \in \mathcal{C}_{p}(\gamma), 0 \leq \gamma<p, p \in \mathbb{N}^{*}$. If $\alpha \in \mathbb{C}, \alpha \neq-2, \alpha \neq \frac{2}{3}$ and

$$
p \geq \frac{|\alpha|-\gamma(|\alpha-1|+1)}{2|\alpha|-|\alpha-1|-1}
$$

then the integral operator

$$
I_{\alpha, 1}^{p}(z)=I_{\alpha}^{p}(z)=\int_{0}^{z} p t^{p-1}\left(\frac{f(t)}{t^{p}}\right)^{1-\frac{1}{\alpha}}\left(\frac{g^{\prime}(t)}{p t^{p-1}}\right)^{\frac{1}{\alpha}} d t
$$

is the p-th power of a univalent function in $\mathcal{U}$.

Letting $\beta=n=1, \gamma_{1}=\gamma, f_{1}=f, g_{1}=g$ and choosing $\alpha_{1}=\alpha=1$ in Theorem 2.2, we have:

Corollary 2.4. Let the functions $f \in \mathcal{S}_{p}^{*}(\gamma)$ and $g \in C_{p}(\gamma), 0 \leq \gamma<p, p \in \mathbb{N}^{*}$, then the integral operator

$$
I_{1,1}^{p}(z)=I^{p}(z)=\int_{0}^{z} g^{\prime}(t) d t
$$

is the p-th power of a univalent function in $\mathcal{U}$. 


\section{References}

Çağlar, M. (2015). The relationships between p-valent functions and univalent functions, Analele Stiitifice ale Universitaii Ovidius Constanta, Seria Matematica.

Deniz, E. (2013). P-subordination chains and p-valence criteria, Journal of Inequalities and Applications, 1, 1-7. https://doi.org/10.1186/1029-242X-2013-127

Deniz, E., Orhan, H., \& Çağlar, M. Sufficient conditions for pv alence of an integral operator (submitted).

Frasin, B. A. (2009). New general integral operators of p-valent functions, Journal of Inequatilies Pure and Applied Mathematies, 10(4), 9 pp.

Hallenbeck, D. J., \& Livingston, A. E. (1975). Subordination chains and p-valent functions, preprint.

Miller, S. S., Mocanu, P. T., \& Reade, M. O. (1978). Starlike integral operator, Pacific J. Math, 79, 157-168. https://doi.org/10.2140/pjm.1978.79.157

Oprea, A., \& Breaz, D. (2015). Univalence conditions for a general integral operator, Analele Stiitifice ale Universitatii Ovidius Constanta, 23(1), 213-224.

Pescar, V. (2000). New criteria for univalence of certain integral operators, Demonstratio Math, 33, 51-54. https://doi.org/10.1515/dema-2000-0107

Pescar, V., \& Owa, S. (2000). Sufficient conditios for univalence of certain integral operators, India J. Math. 42, 347-351.

Seenivasagan, N., \& Breaz, D. (2007). Certain sufficient conditions for univalence, Gen. Math, 15(4), 7-15.

Srivastava, H. M., Deniz, E., \& Orban, H. (2010). Some general univalence criteria for a family of integral operators, Appl. Math. comput, 215, 3696-3701. https://doi.org/10.1016/j.amc.2009.11.007

\section{Copyrights}

Copyright for this article is retained by the author(s), with first publication rights granted to the journal.

This is an open-access article distributed under the terms and conditions of the Creative Commons Attribution license (http://creativecommons.org/licenses/by/4.0/). 